\title{
Deformation Mechanism of the Coal ahead of Fully Mechanized Caving Face under High-Intensity Mining Condition
}

\author{
Can Zhao, ${ }^{1}$ Liang Chen (iD, ${ }^{2}$ Bing Wu, ${ }^{1}$ Jingui Zhang, ${ }^{3}$ Dahe Yan, \\ Yang $\mathrm{Li}^{5}$ and Zhiheng $\mathrm{Cheng}^{5}$ \\ ${ }^{1}$ China University of Mining and Technology-Beijing, Beijing 100083, China \\ ${ }^{2}$ Safety Branch, China Coal Research Institute, Beijing 100013, China \\ ${ }^{3}$ Shenmu Energy Bureau of Shaanxi Province, Shenmu 719300, China \\ ${ }^{4}$ Shaqu No. 1 Mine of Huajin Coking Coal Co., Ltd., Liulin 033300, China \\ ${ }^{5}$ North China Institute of Science and Technology, Beijing 101601, China \\ Correspondence should be addressed to Liang Chen; 3303178898@qq.com
}

Received 17 September 2021; Revised 22 November 2021; Accepted 25 November 2021; Published 16 December 2021

Academic Editor: Qian Chen

Copyright (C) 2021 Can Zhao et al. This is an open access article distributed under the Creative Commons Attribution License, which permits unrestricted use, distribution, and reproduction in any medium, provided the original work is properly cited.

\begin{abstract}
In order to study the coal deformation and failure mechanism in fully mechanized caving face under the high-intensity mining, based on the equivalent mechanical model of transversely isotropic cylindrical coal with fractures, the equivalent equations for axial, radial, and volume strains of coal sample loaded in linear elastic and plastic stages were derived in this paper. The equivalent mechanical model shows good reliability through the conventional triaxial experiment. Taking the N1206 workface in Yuwu coal mine of Luan group as the example, we have simulated the stress concentration factor of the coal body ahead of the working face with FLAC and divided three regions according to stress distribution in coal mining. Mathematical equations were derived to express the horizontal and vertical stress, which provide theoretical guidance of the stress paths in triaxial experiment about real mining stress environment simulation. Experimental results show that the volume strain's value is about $0.4 \%$ in the coal mass deformation progress of axial compression increasing slowly area. In axial compression increasing rapidly area, the volume strain's value varies from $0.41 \%$ to $0.27 \%$, and the radical strain changes from compression deformation to expansion deformation gradually. The volume strain of coal sample increases sharply in axial compression releasing rapidly area; meanwhile, there are good linear relationships between Poisson's ratio and axial strain and radial strain.
\end{abstract}

\section{Introduction}

China's coal seam gas is richly endowed, and its reserves are roughly comparable to those of natural gas. Gas flow in coal seams is a complex process of mutual coupling between gas transport and solid deformation of coal seams [1]. With the further extension of coal mining depth, the pressure gradient of coal seam gas is larger than that of shallow coal seam, and the gas content in coal seam also increases gradually [2], which makes the working face more prone to gas gushing and coal and gas protrusion and other power disaster accidents. Therefore, this coal seam gas has become an important factor to restrict the safe and efficient production of coal mines [3]. Intensive study on the deformation and failure mechanism of coal body ahead of fully mechanized caving face is helpful to make the corresponding fracture and seepage fields of coal body clear [4-6]. It has important theoretical guiding significance and engineering application value for the gas control and coal and gas outburst prevention in high gas working face $[7,8]$.

With the advance of the working face, the front coal body would successively become the original rock stress area, the stress concentration area, and the pressure relief area, where the elastic deformation and plastic deformation gradually occur until the coal-body fracture. At present, the relevant research at home and abroad focuses on the determination of "three areas" of coal body ahead of working face. Xie et al. [9] studied the distribution law of mining-induced stress of 
coal body ahead of fully mechanized caving by establishing a mechanical model of coal body damage in working face. The isotropic damage model established by Hansen and Schreyer $[10,11]$ was used to study the damage characteristics of coal body. Pu and Miao [12] used RFPA software to simulate the distribution characteristics of support pressure ahead of fully mechanized caving face, and the scope of "three zones" of fully mechanized caving working face was divided. Jiang and $\mathrm{Hu}[13,14]$ used the microseismic monitoring system to determine the width of the "three zones" of coal body ahead of the working face. In addition, Su and Huang et al. $[15,16]$ simulated the in situ stress field through deformation and failure tests of coal samples under different stress paths, and the deformation and failure laws of coal body ahead of the working face were also studied. Jianping et al. [17] used a triaxial test platform with gas-contained coal and rock samples to study the transformation law of strain and permeability of coal body under load.

Due to the differences between the loading and unloading paths of triaxial test and the real stress state of coal body ahead of the working face, how to improve the accuracy of the test and then quantitatively judge the deformation of coal body still needs to lucubrate, especially for the deformation characteristics study of coal body ahead of fully mechanized caving face under high-intensity mining condition. Based on equivalent pore-fracture mechanical model of cylindrical coal sample, the stress-strain equation of coal sample when loaded in the plastic stage is derived in this paper. The horizontal stress and vertical stress distributions of coal body in the relief zone, stress concentration zone, and normal pressure zone ahead of fully mechanized caving working face are obtained by using numerical simulation. According to the real working face stress distribution in different stages, the GCTS triaxial instrument is then used to load and unload simultaneously the axial pressure and confining pressure to the coal sample. The deformation characteristics of coal body and the development of fractures under the real stress environment of fully mechanized caving face are emphatically studied. The research results provide a reference for the study of coal and gas solid-gas coupling law, which is of great significance to realize the comining of coal and gas in the high-strength fully mechanized caving face.

\section{Theoretical Analysis of Deformation of Coal Sample under Loading}

As a typical sedimentary rock, coal seam has obvious stratification [18], and this porous medium consists of cracks and the coal matrix which contains a solid skeleton and pores [19]. Considering the coal body as transverse isotropic material and the pores as isotropic material, Xue [20] proposed a pore-crack equivalent mechanical model as shown in Figure 1, and the equivalent radial strain, equivalent axial strain, and equivalent volume strain of coal samples during linear elastic deformation are derived.

In the linear elastic deformation stage, the equivalent displacements $\widetilde{\varepsilon_{x}}, \widetilde{\varepsilon_{y}}$, and $\widetilde{\varepsilon_{z}}$ along $x, y$, and $z$ directions of the equivalent coal and rock mass model would have the relationship shown in the following equation:

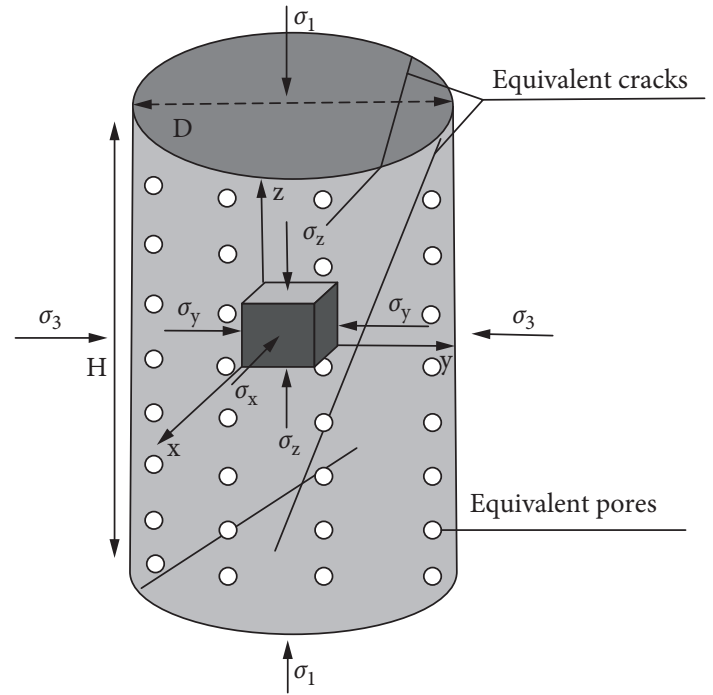

Figure 1: Equivalent mechanical model of the coal containing pores and cracks [20].

$$
\left\{\begin{array}{l}
\widetilde{\varepsilon_{x}}=\frac{\left(\delta_{\phi x}+\delta_{\tau x}+\delta_{o x}\right)}{D}, \\
\widetilde{\varepsilon_{y}}=\frac{\left(\delta_{\phi y}+\delta_{\tau y}+\delta_{o y}\right)}{D}, \\
\widetilde{\varepsilon_{z}}=\frac{\left(\delta_{\phi z}+\delta_{\tau z}+\delta_{o z}\right)}{H},
\end{array}\right.
$$

where $\delta_{\varphi x}, \delta_{\varphi y}$, and $\delta_{\varphi z}$ are the pore displacements of coal samples along $x, y$, and $z$ directions, respectively. $\delta_{\tau x}, \delta_{\tau y}$, and $\delta_{\tau z}$ are the displacements of coal matrix, respectively. $D$ is the diameter of the cylindrical coal sample, $\mathrm{mm} ; H$ is the height of the coal sample, mm.

Therefore, the equivalent volume strain of the media in the elastic stage is

$$
\tilde{\varepsilon_{V}}=\tilde{\varepsilon_{x}}+\widetilde{\varepsilon_{y}}+\widetilde{\varepsilon_{z}} \text {. }
$$

However, in coal's conventional triaxial mechanical test, the coal would experience the compaction stage, elasticity stage, and volume expansion stage from the initial stress loading to the failure, successively. The linear elastic deformation is only a part of the process and further analysis of the subsequent plastic deformation is required.

When the coal-rock mass enters the plastic deformation stage, its strain state is not only related to the loading and unloading stress state but also related to the loading and unloading path, where the plastic constitutive relation between incremental stress and incremental strain can only be established. According to increment theory [19], there must be a small increment in the total strain $d \varepsilon_{i j}$ containing the elastic strain increment $d \varepsilon_{i j}^{e}$ and the plastic strain increment $d \varepsilon_{i j}^{p}$, when a small increment in the external load existed, where $d \varepsilon_{i j}=d \varepsilon_{i j}^{e}+d \varepsilon_{i j}^{p}$. 
According to the characteristics of pore-crack structure, it is considered that the whole deformation of coal body consists of coal matrix deformation, pore deformation, and crack deformation on the surface of coal body. The strain increment at each place in the coal body $d \varepsilon_{i j}$ includes the matrix deformation increment $d \varepsilon_{i j}^{\phi}$, the main fracture deformation increment $d \varepsilon_{i j}^{\tau}$, and the pore deformation increment inside the coal body $d \varepsilon_{i j}^{o}$. Since most of the deformable pores are compacted after the compaction stage of coal and rock mass, the pore deformation at this stage is ignored. Therefore, the plastic stage strain increment includes the matrix deformation increment of coal body and the main fracture deformation increment of coal body.

$$
d \varepsilon_{i j}=d \varepsilon_{i j}^{\phi}+d \varepsilon_{i j}^{\tau} .
$$

In the plastic deformation stage, elastic strain $\varepsilon_{i j}^{\phi e}$ and plastic strain $\varepsilon_{i j}^{\phi p}$ occur in the coal-rock matrix. According to the increment theory,

$$
d \varepsilon_{i j}^{\phi}=d \varepsilon_{i j}^{\phi e}+d \varepsilon_{i j}^{\phi p} .
$$

When elastic deformation occurs in the coal-rock matrix, the generalized Hooke's law is still satisfied. The elastic strain increment along $x, y$, and $z$ directions is

$$
\left\{\begin{array}{l}
d \varepsilon_{x}^{\phi e}=\frac{\left(d \sigma_{3}-v_{x} d \sigma_{1}-v_{x} d \sigma_{3}\right)}{E_{x}} \\
d \varepsilon_{y}^{\phi e}=\frac{\left(d \sigma_{3}-v_{x} d \sigma_{1}-v_{x} d \sigma_{3}\right)}{E_{x}} \\
d \varepsilon_{z}^{\phi e}=\frac{\left(d \sigma_{3}-2 v_{z} d \sigma_{3}\right)}{E_{z}}
\end{array}\right.
$$

The constitutive equations of plastic strain increment along $x, y$, and $z$ directions of coal matrix are obtained by applying the incremental theory when the coal transformed to the plastic strain, as shown in equation (6). Parameter $d \lambda$ is a nonnegative scalar proportional coefficient, which is related to the loading path and $d \lambda=3 d \varepsilon_{i} / 2 d \sigma_{i}$.

$$
\left\{\begin{array}{l}
d \varepsilon_{x}^{\phi p}=\frac{1}{3} d \lambda\left(\sigma_{3}-\sigma_{1}\right), \\
d \varepsilon_{y}^{\phi p}=\frac{1}{3} d \lambda\left(\sigma_{3}-\sigma_{1}\right), \\
d \varepsilon_{z}^{\phi p}=\frac{2}{3} d \lambda\left(\sigma_{1}-\sigma_{3}\right) .
\end{array}\right.
$$

Combined with equations (5) and (6), it can be concluded that the displacements of coal-rock mass matrix along $x, y$, and $z$ directions when it transformed to the plastic stage are

$$
\left\{\begin{array}{l}
d \delta_{x}^{\phi}=\left[\frac{\left(d \sigma_{3}-v_{x} d \sigma_{1}-v_{x} d \sigma_{3}\right)}{\mathrm{E}_{x}}+\frac{1}{3} d \lambda\left(\sigma_{3}-\sigma_{1}\right)\right]\left(D-\sum_{i=1}^{n} \frac{h_{i}}{\sin \theta}\right), \\
d \delta_{y}^{\phi}=\left[\frac{\left(d \sigma_{3}-v_{x} d \sigma_{1}-v_{x} d \sigma_{3}\right)}{\mathrm{E}_{x}}+\frac{1}{3} d \lambda\left(\sigma_{3}-\sigma_{1}\right)\right]\left(D-\sum_{i=1}^{n} \frac{h_{i}}{\sin \theta}\right), \\
d \delta_{z}^{\phi}=\left[\frac{\left(d \sigma_{3}-2 v_{z} d \sigma_{3}\right)}{\mathrm{E}_{z}}+\frac{2}{3} d \lambda\left(\sigma_{1}-\sigma_{3}\right)\right]\left(D-\sum_{i=1}^{n} \frac{h_{i}}{\cos \theta}\right) .
\end{array}\right.
$$


When the coal is in the plastic state, especially in the volume expansion stage, the constitutive equation would be obtained by using the D-P criterion as the yield condition of the coal expansion [20-23], where $\tau$ is the shear stress of coal body, $\sigma_{\mathrm{m}}$ means normal stress, and $\alpha$ and $k$ are the test constants only related to the internal friction angle and cohesion of coal body, as shown in the following equation (8):

$$
\left\{\begin{array}{l}
f\left(\sigma_{m}, \tau\right)=-\tau+\alpha \sigma_{m}^{2}+k \sigma_{m}, \\
\tau=\frac{\sqrt{2}}{3}\left(\sigma_{1}-\sigma_{3}\right), \\
\sigma_{m}=\frac{1}{3}\left(\sigma_{1}+2 \sigma_{3}\right) .
\end{array}\right.
$$

When studying the displacement caused by the crack on the coal surface, the normal stress and shear stress on the equivalent crack surface of the coal body can be obtained according to equation (8). When the coal body is in the plastic state, the nonlinear changes would occur on the normal stiffness, tangential stiffness parallel to the crack, and angular stiffness perpendicular to the crack on the surface of a single crack, and corresponding instantaneous increments are $\mathrm{d} k_{\mathrm{n}}, \mathrm{d} k_{f / /}$, and $\mathrm{d} k_{f \perp}$ respectively. As shown in Figure 2, the displacement increments of multiple groups of cracks along $x, y$, and $z$ directions are

$$
\left\{\begin{array}{l}
d \delta_{x}^{\tau}=0 \\
d \delta_{y}^{\tau}=\sum_{i=1}^{n}\left\{\frac{1}{3} \sigma_{1}\left(\frac{\sqrt{2} \sin \theta h_{i}}{d k_{n}}-\frac{\cos \theta l_{i}}{d k_{f / /}}\right)-\frac{1}{3} \sigma_{3}\left(\frac{\sqrt{2} \sin \theta h_{i}}{d k_{n}}+\frac{2 \cos \theta l_{i}}{d k_{f / /}}\right)\right\} \\
d \delta_{z}^{\tau}=\sum_{i=1}^{n}\left\{\frac{1}{3} \sigma_{1}\left(\frac{\sqrt{2} \cos \theta h_{i}}{d k_{n}}+\frac{\sin \theta l_{i}}{d k_{f / /}}\right)+\frac{1}{3} \sigma_{3}\left(\frac{2 \sin \theta l_{i}}{d k_{f / /}}-\frac{\sqrt{2} \cos \theta h_{i}}{d k_{n}}\right)\right\}
\end{array}\right.
$$

Therefore, the equivalent displacement strains of coal body in the plastic stage along $x, y$, and $z$ directions are

$$
\left\{\begin{array}{l}
\widetilde{d \varepsilon_{x}}=\frac{d \delta_{x}^{\phi}}{D} \\
\widetilde{d \varepsilon_{y}}=\frac{\left(d \delta_{y}^{\phi}+d \delta_{y}^{\tau}\right)}{D} \\
\widetilde{d \varepsilon_{z}}=\frac{\left(d \delta_{z}^{\phi}+d \delta_{z}^{\tau}\right)}{H}
\end{array}\right.
$$

Therefore, the equivalent volume strain of coal in the plastic stage is

$$
\widetilde{d \varepsilon_{V}}=\frac{d \delta_{x}^{\phi}}{D}+\frac{d \delta_{y}^{\phi}+d \delta_{y}^{\tau}}{D}+\frac{d \delta_{z}^{\phi}+d \delta_{z}^{\tau}}{H}
$$

\section{The Verification by Triaxial Mechanical Test of Coal-Rock Mass}

The test coal sample is obtained from the N1206 fully mechanized caving face of No. 3 coal seam, Yuwu coal mine, Luan Group, where the buried depth is $460 \sim 510 \mathrm{~m}$ and the average dip angle is $3 \sim 8^{\circ}$. According to the Specification of Gas Geological Map of No. 3 Coal Seam of Yuwu coal mine [24], the relative gas emission of this mine is
$16.93 \sim 19.55 \mathrm{~m}^{3} / t$, which is a high gas mine. The porosity of coal seam is $4.54 \sim 6.79 \%$, the average bulk density is $1.44 \mathrm{t} / \mathrm{m}^{3}$, the hardness coefficient is $0.45 \sim 0.62$, and the hardness of coal body is soft. Large block coal samples are selected in the coal body ahead of the working face, and the standard size samples ( $\Phi 50 \mathrm{~mm} \times H 100 \mathrm{~mm}$ ) is drilled along the parallel bedding direction. The GCTS triaxial test platform was used to test by the displacement control method. In the period of triaxial tests, the confining pressure was held at $6 \mathrm{MPa}$, and the axial pressure was loaded at the rate of $0.03 \% \mathrm{~mm} / \mathrm{min}$ until failure.

The quality of sample $(\Phi 49.41 \mathrm{~mm} \times H 102 \mathrm{~mm})$ is $274.76 \mathrm{~g}$. The density is $1.40 \mathrm{~g} / \mathrm{cm}^{3}$ and the porosity of the coal sample is $5.58 \%$. As shown in Figure 3, two main cracks can be observed on the cylindrical surface of the coal sample: the length of fissure 1 is $63 \mathrm{~mm}$, extending upward to the upper end face, and fissure 2 is $44 \mathrm{~mm}$, extending downward to the lower end face. Therefore, the two main cracks have a certain influence on the macroscopic mechanical properties of coal samples. At present, there are few studies on the transverse isotropic mechanical parameters of coal samples with cracks. The mechanical test data of different coal bodies are given according to [25]. The rates of elastic modulus and Poisson's ratio along the vertical bedding direction $\left(E_{\perp}\right.$ and $\left.\nu_{\perp}\right)$ and the parallel bedding direction $\left(E_{/ /}\right.$and $\left.v_{/ /}\right)$are $2.5 \sim 7.7$ and 1.8 3.1, respectively. According to the occurrence conditions of No. 3 coal seam, values of rates are 4.8 and 2.1, respectively. It can be seen that $E_{\perp}$ and $E_{/ /}$are $3800 \mathrm{MPa}$ and $792 \mathrm{MPa}$, 


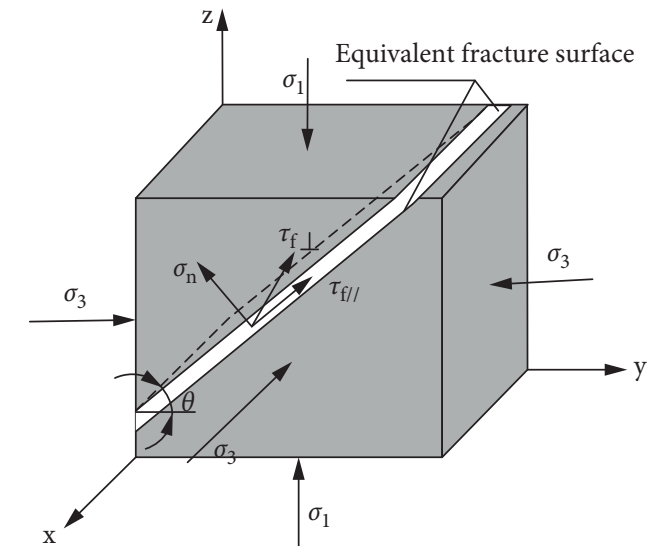

Figure 2: Stress analysis of the coal's fracture surface.
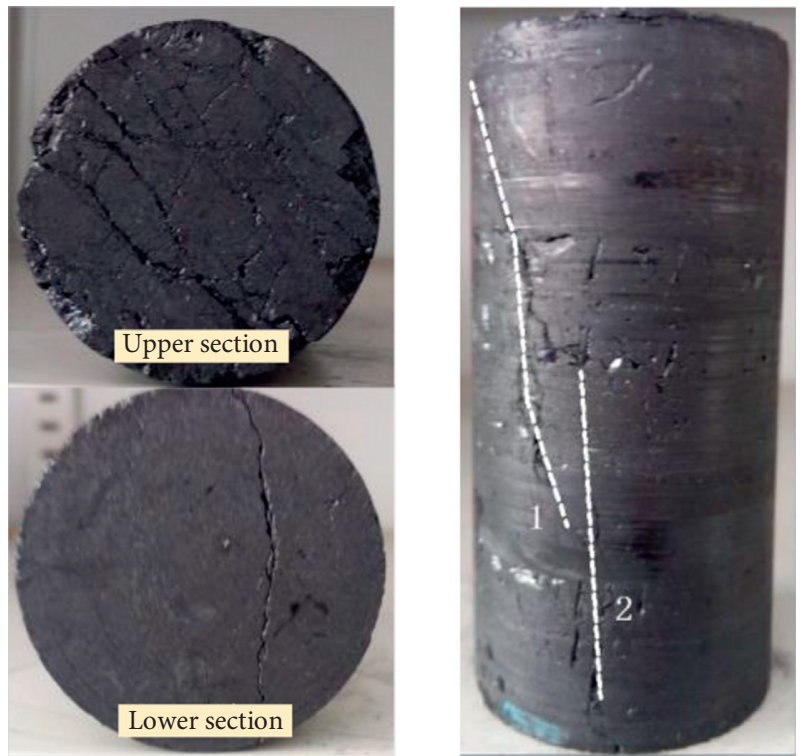

FIGURE 3: Major fracture distribution of coal surface.

respectively, and $\nu_{\perp}$ and $v_{/ /}$are 0.38 and 0.18 , respectively, where the elastic modulus corresponding to coal matrix and coal pore medium is $792 \mathrm{MPa}$ and $2900 \mathrm{MPa}$, respectively, and corresponding Poisson's ratio is 0.32 and 0.18 . The details of surface crack parameters of coal are shown in Table 1. Since displacement control was adopted in this test, the axial compression loading rate was $0.03 \mathrm{~mm} / \mathrm{min}$, and the corresponding average axial stress rate was $0.013 \mathrm{MPa} / \mathrm{min}$. It can be concluded that the plastic deformation coefficient $\mathrm{d} \lambda=0.023$. According to the value of [26], the internal friction angle of the sample is $39.8^{\circ}$ and the average cohesion force is $7.4 \mathrm{MPa}$.

The stress-strain curve obtained from the test is shown in Figure 4. Based on the anisotropic deformation of the coal, it can be considered that the stress and strain of the coal have a linear relationship in the compaction stage and the elastic deformation stage, and, in the yield stage, the stress and strain show nonlinear-correlation relationship when the coal is in the plastic deformation stage, which is consistent with the theoretical analysis results in Section 1. In the failure stage of coal mass, the main failure mode is shear dilatation. The confining pressure is $6 \mathrm{MPa}$ and the axial pressure is in the range of $0 \sim 15 \mathrm{MPa}$. Correspondingly, the axial strain, radial strain, and volume strain are $0 \sim 0.00299,0 \sim-0.00126$, and $0 \sim 0.00048$, respectively, and the sample is in the linear elastic stage. When the axial compression is greater than $15 \mathrm{MPa}$, it belongs to the plastic stage, and the corresponding axial strain is 0.00299-0.00618.

Due to the displacement controlling loading method, the axial strain still changes linearly before the failure. The radial strain and volume strain are $-0.00126 \sim-0.00831$ and $0.00048 \sim-0.01042$, respectively, which are 5.6 and 22.7 times of the anisotropic strain in the linear elastic stage. Figure 5 shows the stress-strain curves of linear elastic stage and plastic stage calculated by theory, which well verifies the axial, radial, and volume strain curves obtained from the tests. Although it is large in the range of isotropic strain, it can be used to reflect the isotropic deformation law of coal samples in the process of triaxial loading and unloading.

Because of the large dispersion of coal and rock and the large variation of different coal seams, the theoretical results described in the previous section are only applicable to the same coal seam. Therefore, we can later select enough coal and rock samples to achieve generalization of the theoretical analysis.

\section{Study on Loading Deformation of Coal Body ahead of Fully Mechanized Caving}

4.1. Numerical Simulation and Theoretical Analysis. The structure of Yuwu coal mine is mostly monoclinal in the direction of south and north. The angle of its wings is less than $10^{\circ}$. 3\# coal seam is located in the lower part of Permian Shanxi Formation, and its buried depth range is 350-642 m with $6 \mathrm{~m}$ thickness coal seam, which is stable coal seam, and its vitrinite reflectance is $2.01 \sim 2.94 \%$ belonging to mainly lean and lean coal. The relative gas emission of this mine is $16.93 \sim 19.55 \mathrm{~m}^{3} / t$, which is a high gas mine. Taking the N1206 working face of the mine as an example, the working face adopts top coal caving and the machine mining height is $3.3 \mathrm{~m}$, while the coal drawing thickness is $3 \mathrm{~m}$. The working face inclination length is $300 \mathrm{~m}$, and the strike length is $1200 \mathrm{~m}$. The daily coal output of this high-intensity mining is $8800 \sim 12000 t$, and the buried depth of coal seam in the area is $480 \mathrm{~m}$. The $109 \mathrm{~m}$ overburden seam, including 18 layers of strata, above the coal seam is selected as the research object, and the top-down lithology of the overburden seam is followed by mudstone $(2.4 \mathrm{~m})$, siltstone $(5.3 \mathrm{~m})$, fine-grained sandstone $(2.2 \mathrm{~m})$, medium grained sandstone $(3.8 \mathrm{~m})$, sandy mudstone $(2.3 \mathrm{~m})$, siltstone $(5.19 \mathrm{~m})$, mudstone $(3.5 \mathrm{~m})$, siltstone $(2.5 \mathrm{~m})$, medium-grained sandstone $(2.5 \mathrm{~m})$, mudstone $(1.4 \mathrm{~m})$, siltstone $(4.1 \mathrm{~m})$ sandy mudstone $(2.8 \mathrm{~m})$, siltstone $(2.6 \mathrm{~m})$ of coarse grained sandstone $(2 \mathrm{~m})$, and the sediment layer, interaction $(50 \mathrm{~m})$. The numerical model of the fully mechanized caving working face was established by FLAC software with $400 \mathrm{~m}$ length ( $x$ 
TABLE 1: Mechanical property parameters of coal's fracture structure.

\begin{tabular}{lccc}
\hline Main fractures & Normal stiffness $K_{n} / \mathrm{MPa}$ & Tangential stiffness $K_{s} / \mathrm{MPa}$ & \multicolumn{2}{c}{ Main fractures distribution } \\
Angles $\left({ }^{\circ}\right)$ & 71 & Widths $(\mathrm{cm})$ \\
\hline Fracture 1 & 3800 & 1200 & 0.15 \\
Fracture 2 & 3800 & 1200 & 84 \\
\hline
\end{tabular}

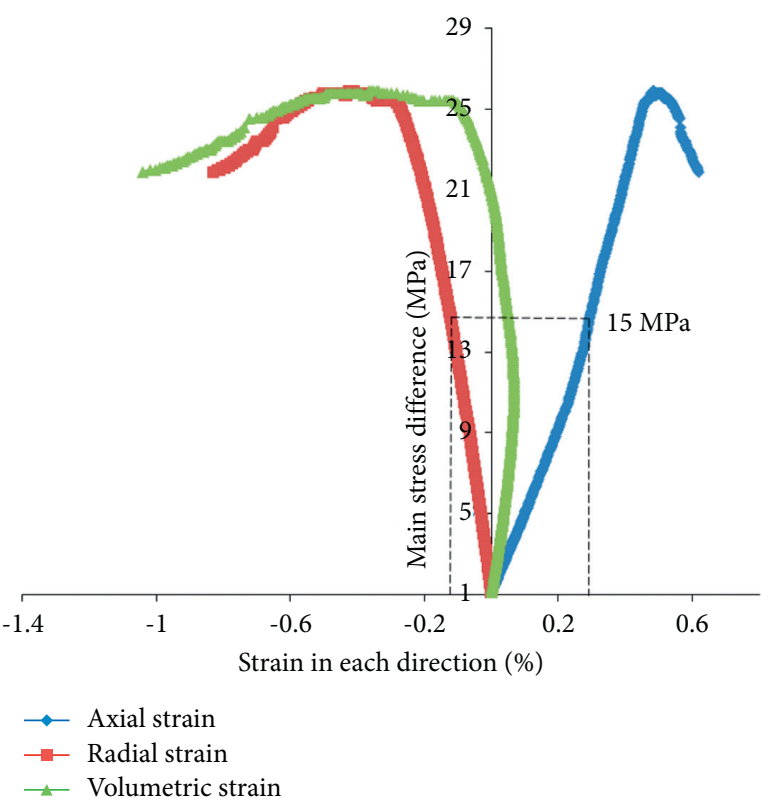

FIgURE 4: Full stress-strain curves of loaded coal sample.

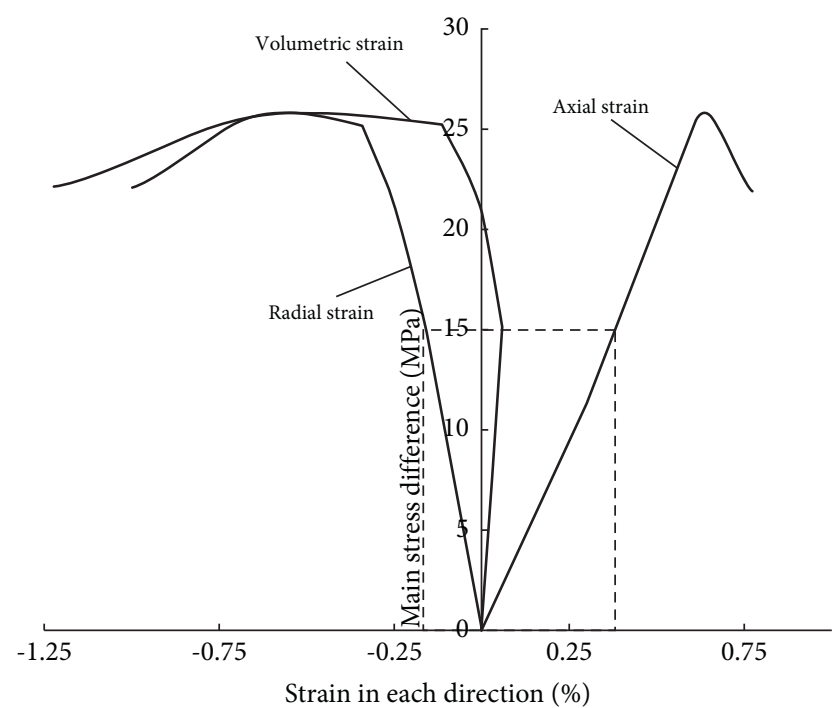

Figure 5: The curves of linear elastic and plastic stage.

direction), $400 \mathrm{~m}$ width ( $y$ direction), and $200 \mathrm{~m}$ height $(z$ direction). The loading stress of the overburden of the model is $8.1 \mathrm{MPa}$, and the failure criterion of coal body is Coulomb mole. Therefore, the stress distribution diagram of the coal body ahead of fully mechanized caving can be obtained, as shown in Figure 6.
Due to the fact that these two roadways have the pressure relief effect on the coal body near the fully mechanized caving working face, the stress will be unloaded at the coal wall near roadways, and the stress concentration phenomenon will not appear when the horizontal stress along $X$-axis applied on the coal body in front of the working face is greater than that of the initial stress; and one side of the working face along the $Y$-axis direction is the coal wall of the working face where the stress is completely unloaded; the other side is the initial stress zone without stress concentration. However, the peak value of stress concentration is smaller than that of vertical stress along the $Z$-axis due to the pressure relief of roadways. With the cylindrical coal sample in this test, stress path in the $Y$-axis and $Z$-axis can be used to guide the loading mode of confining pressure and axial pressure in the test to reflect the reality horizontal stress in the field. According to the horizontal stress expressions of mining dynamics of 3 different typical mining conditions of mining unloading proposed by Xie Heping, the common features of 3 typical mining are refined. Although the peak bearing pressure coefficients and the influence range of the 3 typical mining methods are different, the overall bearing pressure curve ramp-up zone can be further divided into the slowly increasing pressure zone and the sharply increasing pressure zone. Therefore, according to Figure 7, the mining stress on the coal body in front of the working face can be divided into three zones: Rapid step-down zone $\left(L_{1}\right)$, rapid pressurization zone $\left(L_{2}\right)$, and slow pressurization zone $\left(L_{3}\right)$. The results show that the stress concentration coefficient at the peak value of vertical stress concentration is 2.23 , and the horizontal stress concentration coefficient is 0.65 . At the boundary between the slow pressure boost zone and the rapid pressure boost zone, the vertical stress concentration coefficient is 1.16 , and the horizontal stress concentration coefficient is 0.92 . Therefore, the stress distribution characteristics of the fully mechanized caving working face and the axial pressure and confining pressure values corresponding to each critical point in the simulation test can be obtained, as shown in Figure 7.

According to the actual situation of fully mechanized caving working face, the vertical stress of coal body at point $A$ in front of the working face is the residual strength of coal body $R_{\mathrm{C}}$, and the horizontal stress is 0 , as shown in Figure 8 . On the influence of different mining speed and coal seam occurrence conditions, the length of the three zones will change in front of the corresponding fully mechanized caving working face. According to [27], the actual stress distribution curve of the fully mechanized caving face can be simplified as a linear piecewise function of stress and distance. The mining speed of fully mechanized caving face is set as $V$, and the corresponding time of the three zones is 


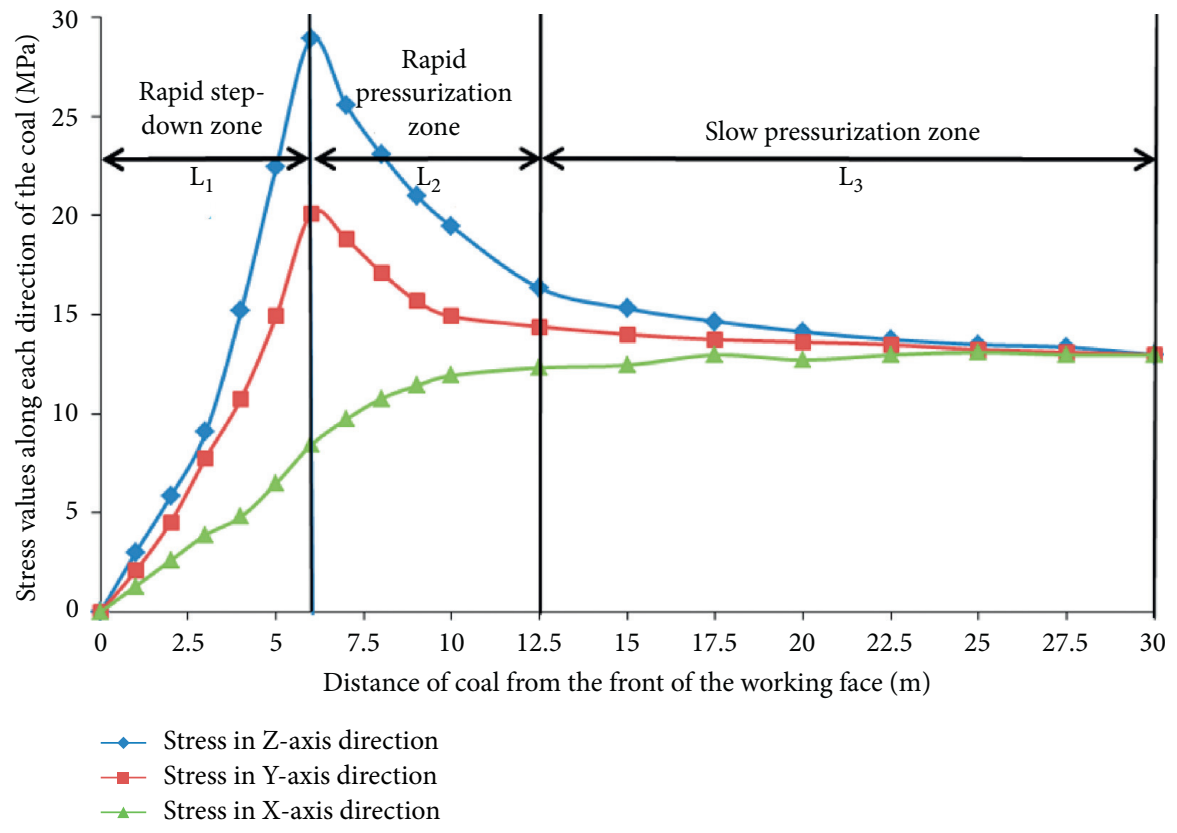

FIgURE 6: Isotropic stress distributions of the coal within $30 \mathrm{~m}$ ahead of working face.

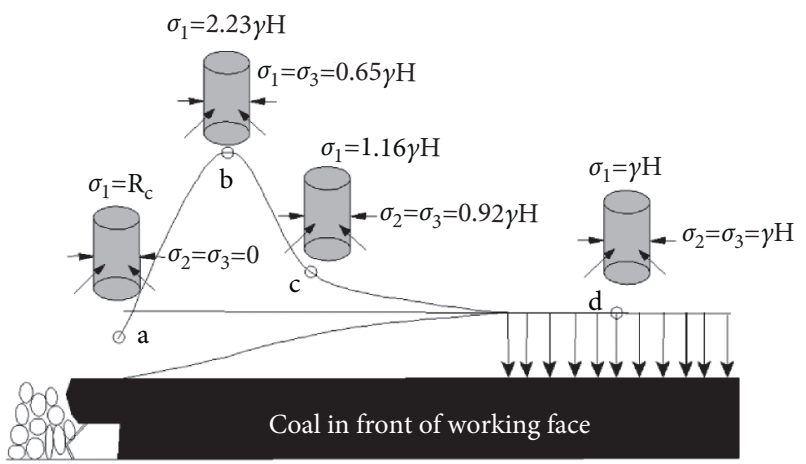

FIGURE 7: Stress distributions of the coal in fully mechanized caving face.

obtained as $L_{1} / V, L_{2} / V$, and $L_{3} / V$, respectively. Given that the slopes of the line in the corresponding interval of vertical stress are $K_{1}, K_{2}$, and $K_{3}$, the corresponding values are $\left(2.23 \gamma H-\mathrm{R}_{\mathrm{c}}\right) / L_{1},-1.07 \gamma H / L_{2}$, and $-0.16 \gamma H / L_{3}$, which correspond to the axial compression loading rates of $\left(\mathrm{R}_{\mathrm{c}}-2.23 \gamma H\right) v, 1.07 \gamma \mathrm{Hv}$, and $0.16 \gamma \mathrm{H} v$ in different stages of

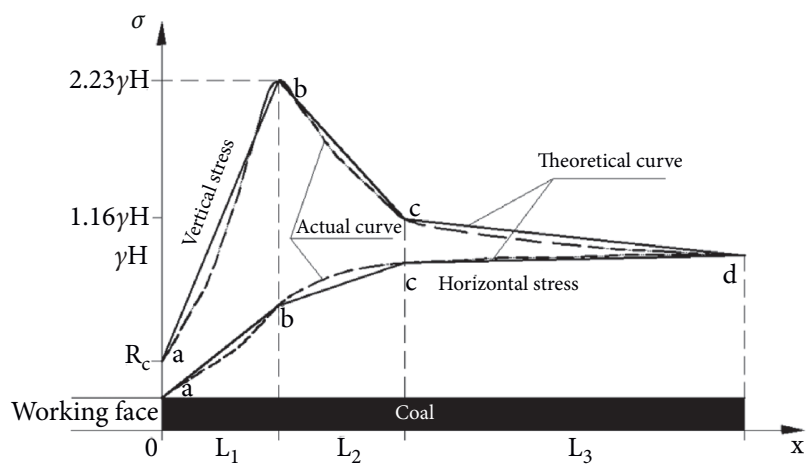

FIGURE 8: The actual stress distribution curve and theoretical curve.

triaxial test, respectively. To ensure that the test is closer to the real stress state of the actual fully mechanized caving face, the loading rates of confining pressure are $-0.65 \gamma H \mathrm{H}$, $-0.27 \gamma H V$, and $-0.08 \gamma H V$, respectively.

Therefore, the vertical stress distribution function can be obtained as follows:

$$
\sigma_{1}= \begin{cases}\frac{2.23 \gamma H-R_{c}}{L_{1}} x+R_{c}, & \left(0 \leq x<L_{1}\right), \\ -\frac{1.07 \gamma H}{L_{2}} x+\frac{1.16 \gamma H L_{2}-0.07 \gamma H L_{1}}{L_{2}}, & \left(L_{1} \leq x<L_{1}+L_{2}\right), \\ -\frac{0.16 \gamma H}{L_{3}} x+\frac{L_{3} \gamma H-\left(L_{1}+L_{2}\right) 0.16 \gamma H}{L_{3}}, & \left(L_{1}+L_{2} \leq x<L_{1}+L_{2}+L_{3}\right) .\end{cases}
$$


The corresponding horizontal stress distribution function is

$$
\sigma_{3}= \begin{cases}\frac{0.65 \gamma H}{L_{1}} x, & \left(0 \leq x<L_{1}\right), \\ \frac{0.27 \gamma H}{L_{2}} x+\frac{0.65 \gamma H L_{2}-0.27 \gamma H L_{1}}{L_{2}}, & \left(L_{1} \leq x<L_{1}+L_{2}\right), \\ \frac{0.08 \gamma H}{L_{3}} x+\frac{0.92 L_{3} \gamma H-\left(L_{1}+L_{2}\right) 0.08 \gamma H}{L_{3}}, & \left(L_{1}+L_{2} \leq x<L_{1}+L_{2}+L_{3}\right) .\end{cases}
$$

4.2. Analysis of Simulation Test Results. The diameter of the test coal sample is $49.31 \mathrm{~mm}$, the height is $100.55 \mathrm{~mm}$, and the mass is $260.62 \mathrm{~g}$. The surface of the sample contains cracks. According to the daily output of N1206 working face $(8800 \sim 12000 t)$, the daily advance would be about $5 \mathrm{~m}$, and the corresponding mining speed is calculated as $0.014 \mathrm{~m} /$ min. The correlation coefficient (original rock stress is $10 \mathrm{MPa}$; the residual strength of coal is $4.7 \mathrm{MPa}$ ) is substituted. In the triaxial simulation test, the axial compression loading rates of the coal body in front of fully mechanized caving are $0.023 \mathrm{MPa} / \mathrm{min}, 0.149 \mathrm{MPa} / \mathrm{min}$, and $-0.246 \mathrm{MPa} / \mathrm{min}$ in the slow pressurization area, rapid pressurization, and rapid unloading area, and the confining pressure unloading rates at corresponding stages are $0.011 \mathrm{MPa} / \mathrm{min}, 0.038 \mathrm{MPa} / \mathrm{min}$, and $0.091 \mathrm{MPa} / \mathrm{min}$, respectively. In the process of the test, the axial pressure and confining pressure of the coal sample were first loaded to the original stress, and then the triaxial loading and unloading test guided by the above test parameters could truly reflect the deformation characteristics of the coal body in the "three zones" in front of the fully mechanized caving working face. The stress-strain-Poisson's ratio variation curve obtained from the test is shown in Figure 9. Subsections I, II, and III, respectively, correspond to slow pressurization area, rapid pressurization, and rapid unloading area.

As seen in Figure 9, the stress-strain curve of the coal sample is consistent with the mechanical deformation characteristics of conventional triaxial mechanical tests. However, it is illuminated that the volume strain of coal body changes little in the slow pressurized region and the rapid pressurized region. In the relief zone, the volume strain shows a sudden increment, which is 6.7 times that of the previous two stages, indicating that the coal deformation in the fully mechanized caving face is mainly volumetric expansion. The trend of different stages is analyzed as follows:

(1) The slow pressurization stage. Due to the initial rock stress, there existed certain axial and radial strains in the coal. In this stage, the ranges axial strain is from 0.12 to $0.37 \%$, and the volume strain decreases slightly from $0.44 \%$ to $0.41 \%$. Poisson's ratio ranges from 0.23 to 0.035 , which indicates that axial compression is the dominant deformation of coal at this stage, and both axial strain and volume strain belong to linear elastic deformation.

(2) Rapid pressurization stage. At this stage, the axial strain is $0.37 \sim 0.51 \%$, volume strain is $0.42 \sim 0.24 \%$, and Poisson's ratio is $0.035 \sim 0.495$. Compared with the previous stage, the volume compression strain in this stage gradually decreases, and the axial deformation is always dominant, but the radial strain turns from compression deformation to expansion deformation. As the axial compression increases rapidly and the confining pressure decreases slowly at this stage, the increase rate of axial strain is greater than that of radial strain, and the volume strain is still a compression deformation, gradually changing from linear elastic deformation to plastic yield deformation.

(3) Rapid pressure relief stage. At this stage, the axial strain is $0.51 \sim 0.59 \%$, the volume strain is $0.24 \% \sim-0.037$, and Poisson's ratio is $0.035 \sim 0.495$. At this stage, the coal mass undergoes plastic yield to macroscopic failure. When the volume strain is -0.018 , the volume of the sample expands sharply, and the maximum expansion rate is $3.7 \%$. Both volume strain and radial strain are dominated by expansion deformation, and the corresponding expansion velocity increases firstly and then decreases. Due to the rapid unloading of the confining pressure to 0 at this stage, the internal cracks in the coal gradually expanded and coalesced until failure, and the axial pressure of the coal rapidly decreased to the residual strength of $4.7 \mathrm{MPa}$. The absolute value of the change slope of Poisson's ratio changes from less than 1 to greater than 1, indicating that the failure mode of coal samples changes from compression shear failure to dilatancy failure [28]. In addition, it can be concluded that Poisson's ratio has a good linear relationship with the axial strain and radial strain at this stage, as shown in Figure 10. The corresponding expressions are as follows: $\nu=-1.7794, \varepsilon_{\text {radial }}+0.0044 ; \nu=12.505, \varepsilon_{\text {axial }}-6.5166$. It shows that the axial strain and radial strain in this stage also have a linear relationship, which indirectly proves that the displacement along the shear plane occurs when the macroscopic failure of the coal gradually occurs. 


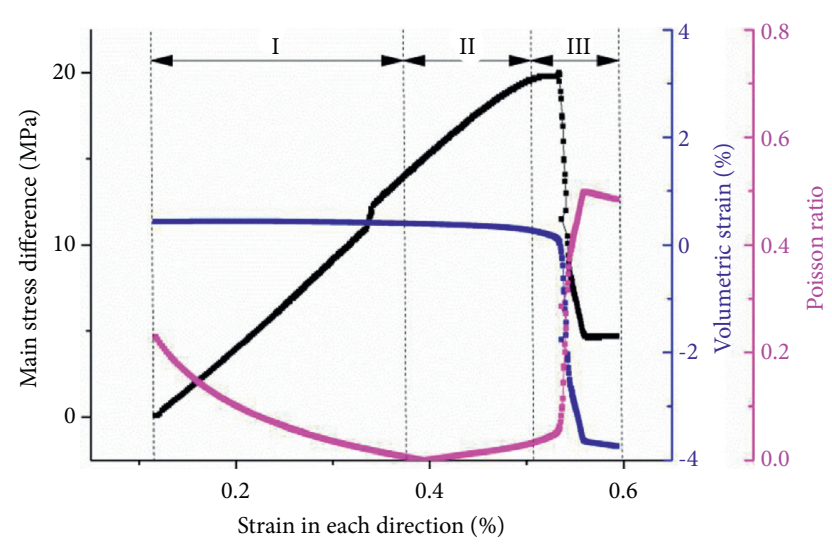

FIGURE 9: The stress-strain-Poisson's ratio curves of coal sample in loading and unloading process.

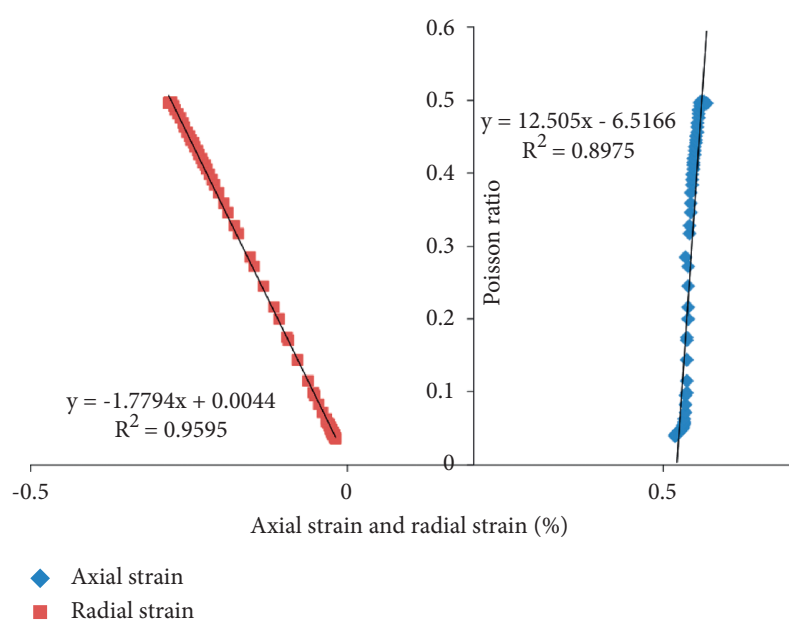

Figure 10: Fitting curves among Poisson's ratio and axial strain and radial strain.

\section{Conclusions}

(1) The equivalent pore-crack model of transversely isotropic coal pointed out that the plastic stage mainly consists of surface crack deformation and coal matrix deformation, leading to the equivalent axial strain, radial strain, and volume strain equations of coal in the plastic stage under loading condition. The conventional triaxial test proves that this theory can be used to characterize the deformation characteristics of coal.

(2) Taking the N1206 working face of Yuwu coal mine in Luan as an example, the stress concentration coefficient of the coal body in front of the fully mechanized caving working face is 2.23 with the consideration of mining intensity through numerical simulation. The coal body in front of fully mechanized caving is divided into slow pressurization area, rapid pressurization, and rapid unloading area, and the expressions of vertical stress and horizontal stress in corresponding stages are derived.
(3) The mining disturbance characteristics of highly intensive fully mechanized caving working face, gained from theoretically analyses, are used to guide the triaxial loading and unloading simulation test, and it is concluded that the axial deformation is dominant in the slow pressurization area. The radial deformation is dominant in the rapidly pressurized region, and the compression deformation turns to the expansion deformation in radial direction. In the rapid unloading area, Poisson's ratio of the coal sample has a linear relationship with the axial strain and radial strain. With the volume expanding rapidly, the internal cracks gradually expand and connect until the failure occurs. When the volume strain is 0.018 , the volume deformation increases sharply, and the failure mode changes from compression shear failure to dilatancy failure.

(4) In this paper, further study is required due to the fact that the influence of gas adsorption and absorption in the process of pressurization and unloading was not considered in the derivation of coal deformation. In addition, considering the large dispersion of raw coal, different locations and larger number of samples should be manufactured in order to make more generalized results.

\section{Data Availability}

The data used to support the findings of this study are available from the corresponding author upon request.

\section{Conflicts of Interest}

The authors report no potential conflicts of interest.

\section{Acknowledgments}

This work was financially supported by the National Natural Science Foundation of China (52074120), Program for Science \& Technology Innovation Talents in Universities of Henan Province (19HASTIT047), and Science and Technology Project of Henan Province (182102310012). This support is gratefully acknowledged.

\section{References}

[1] W. Qin, J. Xu, and X. Peng, "Experimental study of coupling between solid and gas during drainage by advance pressure relief in mining-coal seam," Journal of China University of MiningぬTechnolo-gy, vol. 41, no. 6, pp. 900-905, 2012.

[2] G. Hu, J. Xu, H. Wang, Z. Jin, and R. Wu, "Research on a dynamically coupled deformation and gas flow model applied to low-permeability coal," Journal of China University of Mining\&Technolo-gy, vol. 40, no. 1, pp. 1-6, 2011.

[3] G. Z. Yin, B. He, M. H. Li, J. Cao, H. Qin, and W. P. Li, "Coupling mechanism between flow rate of gas drainage and coal seam abutment stress under mining conditions," Journal of China Coal Society, vol. 40, no. 4, pp. 736-741, 2015.

[4] Y. Xue, J. Liu, P. G. Ranjithc, X. Lianga, and S. Wanga, "Investigation of the influence of gas fracturing on fracturing characteristics of coal mass and gas extraction efficiency based 
on a multi-physical field model," Journal of Petroleum Science and Engineering, vol. 206, Article ID 109018, 2021.

[5] Q. Zou, H. Liu, Y. Zhang, Q. Li, J. Fu, and Q. Hu, "Rationality evaluation of production deployment of outburst-prone coal mines: a case study of nantong coal mine in Chongqing, China," Safety Science, vol. 122, Article ID 104515, 2020.

[6] Q. Zou, H. Liu, Z. Jiang, and X. Wu, "Gas flow laws in coal subjected to hydraulic slotting and a prediction model for its permeability-enhancing effect," Energy Sources, Part A: Recovery, Utilization, and Environmental Effects, pp. 1-15, 2021.

[7] X. He, Rheological Dynamics of Coal Rock Containing Gas, China University of mining and technology press, Xuzhou, China, 1995.

[8] L. Yuan, "Theory of press-relieved gas extraction and technique system of integrated coal production and gas extraction," Journal of China Coal Society, vol. 36, no. 7, pp. 1067-1074, 2011.

[9] Z. Chen and H. Xie, "Damage mechanics analysis on the distribution of abutment pressure around a coal face," Journal China of Rock Mechanics and Engineering, vol. 19, no. 4, pp. 436-439, 2000.

[10] N. R. Hansen and H. L. Schreyer, "A thermodynamically consistent framework for theories of elastoplasticity coupled with damage," International Journal of Solids and Structures, vol. 31, no. 3, pp. 359-389, 1994.

[11] M. R. Salari, S. Saeb, K. J. Willam, S. J. Patchet, and R. C. Carrasco, "A coupled elastoplastic damage model for geomaterrials," Computer Methods in Applied Mechanics and Engineering, vol. 193, no. 27-29, pp. 2625-2643, 2004.

[12] H. Pu and X. Miao, "Numerical simulation of dynamic falling of overlying rocks and abutment pressure in surrounding rocks for fuly-mechanized top-coal caving stope," Journal China of Rock Mechanics and Engineering, vol. 19, no. 4, pp. 3274-3280, 2000.

[13] H. Shi and F. Jiang, "Study on abutment pressure rule of overlying strata spatial structures based on micro-seismic monitoring," Journal China of Rock Mechanics and Engineering, vol. 27, no. S1, pp. 436-439, 2008.

[14] G. Hu, Study on the Characteristics of Strata Behavior and Roof Control in the Large Mining Height Fully-Machanized Face, Taiyuan university of technology, Taiyuan, China, 2006.

[15] C. Su, B. Gao, H. Nan, and X. Li, "Experimental study on acoustic emission characteristics during deformation and failure processes of coal samples under different stress paths," Journal China of Rock Mechanics and Engineering, vol. 28, no. 4, pp. 757-766, 2009.

[16] G. Yin, Q. Huang, and D. Zhang, “Test study of ags seepage characistics of gas-bearing coal specimen during process of deformation and failure in geostress field," Journal China of Rock Mechanics and Engineering, vol. 29, no. 2, pp. 336-343, 2010.

[17] W. Jianping, W. Dengke, and W. Le, "Comparison of permeability between two kinds of loaded coal containing gas samples," Journal of China Coal Society, vol. 29, no. 2, pp. 336-343, 2010.

[18] S. Zhou and B. Lin, The Theory of Gas Flow and Storage in Coal Seams, Coal Industry Publishing House, Beijing, China, 1999.

[19] Z. Pan and L. D. Connell, "Modelling permeability for coal reservoirs: a review of analytical models and testing data: a review of analytical models and testing data," International Journal of Coal Geology, vol. 92, no. 1, pp. 1-44, 2012.

[20] D. Xue, Research on Mechanism of Enhanced Gas Permeability of Coal and Rock under Various Mining Layouts, China
University of Mining and technology (Beijing), Beijing, China, 2013.

[21] S. Liu and T. Zhang, Basic Theory of Elasticity and Plasticity, Huazhong University of Science and Technology, Wuhan, China, press, 2002.

[22] J. Zheng, The Correlation between Mining Distressing Law and Gas Migration Ahead of Working Face, China University of Mining and technology (Beijing), Beijing, China, 2015.

[23] J. Chen, C. Yang, and Y. Guo, "Study of sealability of caven for natural gas storage in deep salt formation based on compaction-dilatancy boundary of salt," Chinese Journal of Rock Mechanics and Engineering, vol. 28, no. S2, pp. 3302-3308, 2009.

[24] X. Zhao, Q. Zhang, and G. Dong, Gas Geological Map Instruction of 3\#coal Seam in Yuwu Coal Mine, China Coal technology Engineering Group Chongqing Research Institute, Chongqing, China, 2010.

[25] T. Li, X. Wu, and H. Tu, "The measurement and analysis on the mechanical property of coal seam," Chinese Journal of Rock Mechanics and Engineering, vol. 35, no. 2, pp. 328-336, 2013.

[26] C. Su, B. Guo, and X. Tang, "Research on acoustic emission characteristics of Zhangcun coal samples in two sizes subject to uniaxial compression," Journal of China Coal Society, vol. 4, no. S1, pp. 12-18, 2013.

[27] D. Xue, H. Zhou, X. Tang, and Y. Zhao, "Mechanism of deformation-induced damage and gas permeability enhancement of coal under typical mining layouts," Chinese Journal of Geotechnical Engineering, vol. 35, no. 2, pp. 328336, 2013.

[28] Q. Huang, Research on Mechanical Characteristics of GasBearing Coal under the Condition of Unloading Confining Pressure, Chongqing university, Chongqing, China, 2011. 